\title{
Detection of Volcanic Plumes by GPS: the 23 November 2013 Episode on Mt. Etna
}

\author{
Massimo Aranzulla ${ }^{1 *}$, Flavio Cannavò $^{1}$, Simona Scollo $^{1}$ \\ 1 Istituto Nazionale di Geofisica e Vulcanologia, \\ Osservatorio Etneo, Sezione di Catania, Italy \\ *massimo.aranzulla@ingv.it
}

\begin{abstract}
The detection of volcanic plumes produced during explosive eruptions is important to improve our under-standing on dispersal processes and reduce risks to aviation operations. The ability of Global Position-ing System (GPS) to retrieve volcanic plumes is one of the new challenges of the last years in volcanic plume detection. In this work, we analyze the Signal to Noise Ratio (SNR) data from 21 permanent stations of the GPS network of the Istituto Nazionale di Geofisica e Vulcanologia, Osservatorio Etneo, that are located on the Mt. Etna (Italy) flanks. Being one of the most explosive events since 2011, the eruption of November 23, 2013 was chosen as a test-case. Results show some variations in the SNR data that can be correlated with the presence of an ash-laden plume in the atmosphere. Benefits and limitations of the method are highlighted.
\end{abstract}

\section{INTRODUCTION}

$\mathrm{M}$ t. Etna, in Italy, has been very active in the last years. Volcanic ash often causes problems to aviation operations, forcing the closure of the International airport in Catania [Scollo et al., 2009]. Since 2011 powerful lava fountain events have generated volcanic plumes that rose up to several kilometres above sea level. Those episodes were characterized by an emergent phase of strombolian activity, a paroxysmal phase, where an eruption column was produced, and a final phase characterized by a reduction in volcanic tremor. Details of the style and type of this activity can be found in Behncke et al. [2014].

In order to reduce the impact that these volcanic plumes have on the local population, the existing monitoring and forecasting system
[Scollo et al., 2009] of the Istituto Nazionale di Geofisica e Vulcanologia, Osservatorio Etneo (INGV-OE) has been improved. A Light Detection and Ranging (LiDAR) system was able to retrieve important features of the volcanic plumes [Scollo et al., 2012] and a doppler radar installed in the Montagnola site, a few kilometres from the volcanic vent [Gouhier et al., 2011] detected the beginning and the end of the paroxysmal phases. Furthermore, a recent study carried out on the video-surveillance system of INGV-OE allowed estimating the column height for several eruptive events occurring between 2011 and 2013 [Scollo et al., 2014]. The presence of the volcanic plume may also be detected by the analysis of the Global Positioning System (GPS) signal. Houlie et al. [2005a] found anomalous values in the ionosphere-free phase measurements (LC) during the 2000 Miyakejima eruption in Japan and 
correlated these values to the path delay effects caused by the volcanic plume. Similar results were found for the eruption of 9 March 2005 of Mt. St. Helens [Houlie et al. 2005b]. The ability of GPS to detect volcanic plumes was also investigated by Aranzulla et al. [2013]. The authors, on the base of satellite images and deposit data, created a model of volcanic plume dispersal in order to evaluate the GPS satellitestation couples which crossed the plume. The GPS data coming from the Etna network were hence processed using the software developed by Massachusetts Institute of Technology (namely GAMIT) [Herring, 2010]. Using the undifferenced post-fit phase residuals, robust cross-statistics were applied to assert the plume detection by GPS signals. Aranzulla et al. [2013] suggested that the GPS network at Etna was able to detect the volcanic plume formed during the eruption of 4-5 September 2007.

Recently, Larson [2013] proposed a new method that analyzes the GPS signal collected during the 2008 and 2009 eruptions of the Okmok and Mt. Redoubt volcanoes and demonstrated that the Signal to Noise Ratio (SNR) data collected by GPS receivers can be used to detect volcanic plumes. SNR is a measurement that compares the level of a given signal with the level of the background noise. It is defined as the ratio of the signal power to the noise power in a given bandwidth, usually expressed in units of decibels/milliwatt $(\mathrm{dBm})$ or decibel/watts $(\mathrm{dbW})$, where a value greater than zero indicates more signal than noise. Finally, Fournier and Jolly [2014] used both the aforementioned methods to highlight the potential of GPS data analysis to improve our understanding of eruptive sequences during the August 6, 2012 Te Maari eruption, Tongariro, New Zealand, where visual observations were limited. All these papers demonstrate that GPS could play an important role in volcanic plume detection. However, new studies and further investigations are necessary. In this paper, we test the method proposed by Larson [2013] to one of the most powerful events occurring at Etna volcano since 2011: the 23 November 2013 lava fountain episode. We first explain the method and describe some potential improvements. We go on to briefly describe the eruption of 23 November 2013, show the main results analyzing the SNR data that are recorded in the binary data (RAW) by the GPS receivers and translated for users as Receiver Independent Exchange Format (RINEX), namely the data interchange format for raw satellite navigation system data. Finally, we discuss the advantages of the proposed approach and the future improvements.

\section{METHODS}

GPS data, i.e. the carrier phase, the code observables and the SNR data, are recorded in a raw binary file that has to be translated into an ASCII RINEX file [Gurtner and Mader, 1990]. The SNR data provide no information about the distance between the satellite transmitting the signal and the receiver, and thus make no direct contribution to positioning solutions. The SNR data are reported as two observable types, named S1 and S2, that refer to the signal strengths for L1 (signal at $1.5754 \mathrm{GHz}$ ) and L2 (signal at $1.2276 \mathrm{GHz}$ ) phase observations. As said, the SNR is defined as the ratio of the carrier to noise density $(\mathrm{C} / \mathrm{N} 0)$ of signal expressed in decibel-Hertz $(\mathrm{dB}-\mathrm{Hz})$ in a given bandwidth (B), usually expressed in decibel. The GPS receiver manufacturers report SNR values assuming $1 \mathrm{~Hz}$ band-width, or $\mathrm{dB}-\mathrm{Hz}$. A detailed description of the different SNR data types can be found in Joseph [2010] and Larson [2013].

Figure 1 shows the SNR data for L1-1.5754 $\mathrm{GHz}$ (blue) and L2-1.2276 GHz (black) relevant to the station EIIV (Figure 2) and satellite 01, 
on 20 November 2013, without volcanic activity.

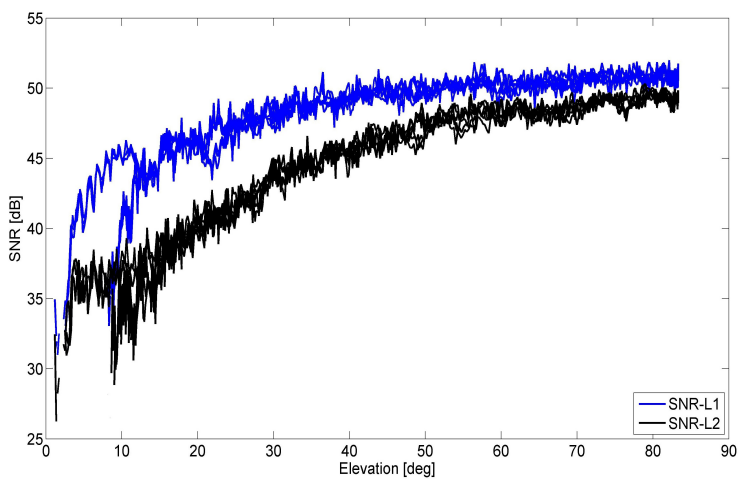

Figure 1: 20 November 2013 L1 and L2 SNR data derived from the EIIV station and satellite 01

As highlighted in Figure 1, the SNR data slowly increase when the satellite rises in elevation angle reaching a better precision at higher elevation angles, mainly caused by surface reflections [Larson, 2013]. The L2-SNR data are used with the aim of detecting the effect of plumes on the signal [Larson, 2013].

In our work, we analyzed data coming from Etn@net, the GPS etnean network of INGV-OE that was installed in 2000 and was upgraded in the following years [Palano et al., 2010]. Today's Etn@net is made up of 42 GPS permanent stations that provide a dense cover of most areas of the volcano edifice. During this study, we analyzed at 30-s sampling rate on a daily basis seven days across the fountain event of 23 November 2013, with data from three days before to three days after the eruption. Of all the 42 GPS stations, 21 GPS receivers were available to study the plume interaction with the GPS signal (Figure 2).

For each investigated day of the year (DOY), the SNR, azimuth and elevation files of each GPS receiver were generated. In order to compare the results, a decimation interval of 30 seconds was applied to the station having a sampling rate of $1 \mathrm{~Hz}$. Furthermore, at this stage we are studying the perturbation only on the GPS signal; consequently, the GLObal NAvigation Satellite System (GLONASS) observations were removed in all the receiver data.

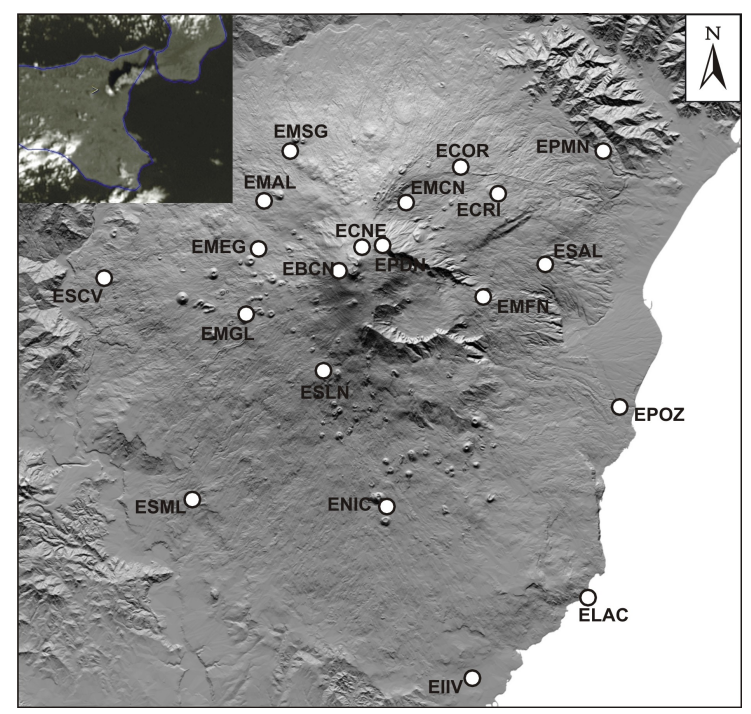

Figure 2: Map of GPS sites with underlying digital elevation model of Etna. Inset, the EUMETSAT Meteosat images of the 23 November 2013 plume at 10:15 GMT

The SNR data with higher elevation angle were used for plume sensing, excluding data below $30^{\circ}$ because of their oscillations caused by ground reflections. The repeatability of the SNR data during the six days across the lava fountain event is similar to the black line in Figure 1. In order to examine and assess the potential to improve the Larson [2013] method, take account of all available satellite tracks, avoid data shift and obtain the same satellite geometry (sidereal day), we used the whole data set of seven days. During the three days before and after the event, we assume that no anomaly disrupted the SNR-L2 signal. Hence, for each receiver-satellite pair, the best fitting curve of the SNR-L2 (elevation) data was computed. This curve represents the SNR-L2 mod$\mathrm{el}$ as a function of the elevation angle and al- 
ANNALS OF GEOPHYSICS, Fast Track 2, 2014

lows us to compute the SNR-L2 at any arbitrary value (i.e. expected value). The difference between the measured and the expected SNRL2 values as a function of epoch for each station and all the satellites were calculated. This procedure allows highlighting anomalies that could be related to the presence of volcanic plume. We also adopted a more general approach to discriminate the different behaviour of SNR in the presence of a volcanic plume. We modelled the SNR as a random variable function of the azimuth and elevation of the satellite, thus taking into account the SNR azimuth anisotropy. We used the non-parametric method introduced by Rosenblatt [1956] and Parzen [1962] that estimates the probability density function of the SNR variable for azimuth/elevation pair values. For that estimation, we considered all the SNR values measured in the 3 days before and after the volcanic activity and, given an azimuth/elevation pair value we considered a range of $5^{\circ} / 3^{\circ}$ for the azimuth/elevation, respectively. Hence, we calculated the probabilities of each measured SNR value during the day of eruption. Since an anomalous SNR should show a low probability of occurrence, in order to emphasize the anomalies from the background, we calculated and plotted the inverse of such a probability.

\section{The 23 November 2013 LAVA Fountain}

The 23 November 2013 eruption was the 42nd event since 2011 and the 17th that had occurred in 2013 from the New South East Crater (NSEC). The eruptive activity began late afternoon the day before with strombolian activity that increased at about 7:00 on 23 November (all timed are GMT). The paroxysmal phase occurred between 9:30 and 10:20. A high eruption column formed and volcanic ash was dispersed toward the North-East/East-North-East direction. The activity ceased at around 11:00 and may be considered one of the most violent events since 2011. A volcanic plume was clearly visible from 9:30 and persisted over eastern Sicily up to 10:30 as observed by EUMETSAT Meteosat satellite data (freely accessible from the EUMETSAT Meteosat web-site).

\section{Results}

We evaluated the SNR-L2 data from 21 GPS stations for the 23 November lava fountain. The station clearly affected by the volcanic plume was EPDN, because of its location North-East of the summit. Figure 3 shows the SNR-L1(elevation) and SNR-L2(elevation) values referred to the EPDN station and satellite 16 for all the 7 studied days. Light red and light blue points represent the SNR L1 and L2 data of the three days before and after the eruptive event; dark red and dark blue points are the values obtained on 23 November 2013. Light points show a similar behaviour on all days without a plume, dark points instead, show anomalies between $40^{\circ}$ and $50^{\circ}$ in elevation. Figure $4 \mathrm{~A}$ shows the difference between the time series of the measured and the expected SNR-L2 values for the EPDN station and for all the satellites linked during the day of the eruption with respect to the epoch.

The red vertical lines represent the beginning and end of the 23 November 2013 event. The yellow lines represent the signal power at \pm 3 $\mathrm{dB}$, where the SNR power is half of its value. The plot shows that some SNR values lie outside this band and this only happens during the eruption time. This is confirmed by the plot of inverse probability (Figure 4B), where the values show an anomalous behaviour for the same station (EPDN) and again during the time eruption.

This anomaly highlights that the SNR values, measured during the eruption, diverge strongly from values observed when there was no activity. Although some other stations show weak anomalies in the SNR-L2 data, this beha- 
viour is not as evident as at EPDN. However, this could be due to the location of the EPDN station, $2.5 \mathrm{~km}$ far away from the volcanic vent and downwind of the volcanic plume dispersal (Figure 1).

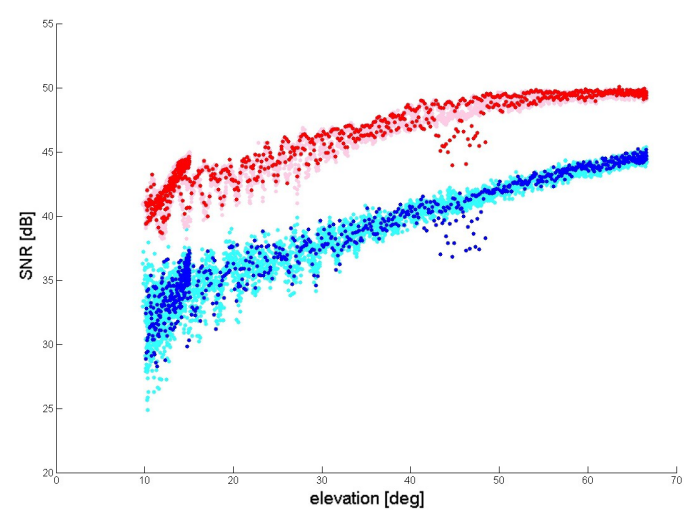

Figure 3: The L1 and L2 SNR data referring to the EPDN station and satellite 16. Light red and light blue points represent the SNR-L1 and SNR-L2 data of the three days before and after the eruptive event; dark red and dark blue points are the values obtained on 23 November 2013 for L1 and L2

We also note that the density of volcanic plume plays an important role on the attenuation of the SNR. Higher volcanic plume density is expected near the vent, and EPDN is one of the closest stations.

\section{DisCUSSION AND CONCLUSIONS}

The SNR signal shows important features that can be used in volcanic plume detection because the SNR can directly measure signal attenuations or blockages. It is worth noting that the 23 November 2013 lava fountain was one of the biggest event since 2011. The strong effects on the EPDN station and the weaker impact on the other close GPS stations suggest that this method might fail during less intense explosive activities. It is well known that Etna has a wide spectrum of explosive activities ranging from strombolian activities to powerful sub-plinian eruptions. This makes Etna a good test site to analyze how the variation of volcanic plume density can affect the SNR signals.

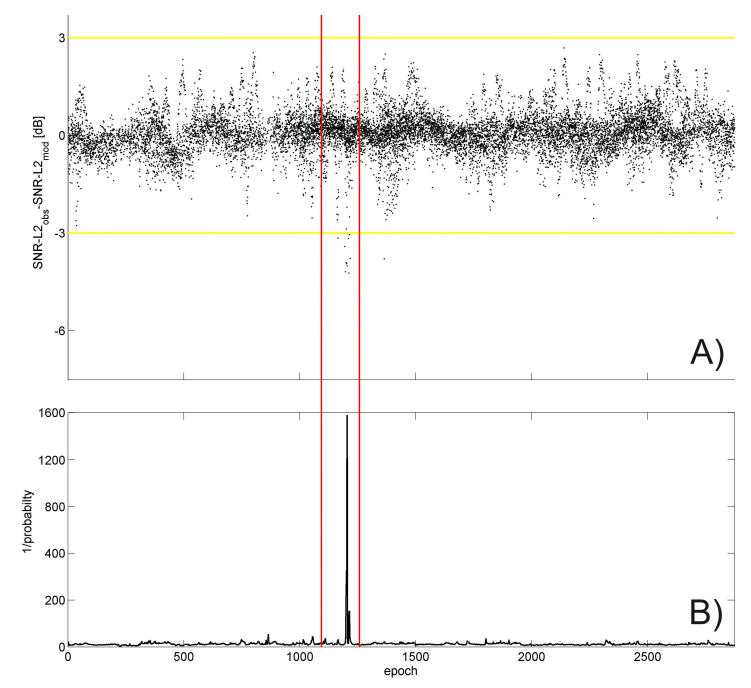

Figure 4: A) The SNR-L2 difference between the measured and the expected SNR-L2 values referring to the EPDN station and all its linked satellites. Black points represent the SNR-L2 data, yellow lines are the $\pm 3 d B$ limit and red vertical lines represent the beginning and end of the 23 November 2013 event. B) Inverse of the estimated probability of the SNR occurrence

In the future a third frequency L5 on the new generation of GPS satellites (blocks IIF and III) and the corresponding S5 observables, could improve the capability to observe volcanic plume. In conclusion, during the 23 November 2013 eruption, the SNR signal showed the presence of volcanic ash. We found that the strength of the SNR signal variation depends on the distance of the station from the volcanic vent. The nearest station clearly shows higher SNR variations. For these reasons, where a GPS network is present, a monitoring of SNR in near real time could be coupled to the other systems, for volcanic cloud detection. 
ANNALS OF GEOPHYSICS, Fast Track 2, 2014

ACKNOWLEDGEMENTS

This work was funded by the MED-SUV project under grant agreement No. 308665 and by the European project APHORISM.

\section{REFERENCES}

[Aranzulla et al., 2012] Aranzulla, M., Cannavò, F., Scollo, S., Puglisi, G., Immè, G. (2013). Volcanic ash detection by GPS signal, GPS Solutions, doi:10.1007/s10291-012-0294-4， 17, 485-497.

[Behncke et al., 2014] Behncke, B., Branca, S. et al. (2014). The 2011-2012 summit activity of Mount Etna: Birth, growth and products of the new SE crater. Journal of Volcanology Geothermal Research, 270, 10-21.

[Gouhier et al., 2011] Gouhier, F., Harris, A. et al. (2011). Lava discharge during Etna's January 2011 fire fountain tracked using MSGSEVIRI, Bull Volcanol, doi: 10.1007/s00445011-0572-y.

[Fournier and Jolly, 2014] Fournier, N., Jolly, A.D. (2014). Detecting complex eruption sequence and directionality from high-rate geodetic observations: The August 6, 2012 Te Maari eruption, Tongariro., J. Volcanol. Geotherm. Res., 286, 387-396.

[Gurtner and Mader, 1990] Gurtner, W., Mader, G. (1990). Receiver independent exchange format version 2, GPS bulletin, 3:1-8.

[Herring et al., 2010] Herring, T.A., King, R.W., McClusky, S.C. (2010). Gamit referencemanual. Department of Earth, Atmospheric, and Planetary Sciences Massachusetts Insti-tute of Technology, Cambridge.

[Houlie et al., 2005a] Houlie N., P. Briole, A. Nercessian, and Murakami, M. (2005a). Sounding the plume of the 18 August 2000 eruption of Miyakejima volcano using GPS, Geop.Res.Let.32, doi:10.1029/2004GL021728.

[Houlie et al., 2005b] Houlie N., P. Briole, A. Nercessian, and Murakami M. (2005b). Vol- canic plume above Mount St. Helens detected with GPS, Eos Trans AGU 86:277. doi:10.1029/2005EO300001.

[Larson, 2013] Larson, K.M. (2013). A new way to detect volcanic plumes, Geophys Res Lett 40. doi: 10.1002/grl.50556, 2013.

[Parzen, 1962] Parzen, E. (1962). On estima-tion of a probability density function and mode, The annals of mathematical statistics, 33(3), 1065-1076.

[Palano et al., 2010] Palano, M., Rossi, M. et al. (2010), Etn@ref: a geodetic reference frame for mt. etna gps networks, Ann Geophys-Italy, 53:49-57, 2010. URL doi:10.4401/ag-4879.

[Rosenblatt, 1956] Rosenblatt, M. (1956). Remarks on some nonparametric estimates of a density function, The Annals of Mathematical Statistics, 27(3), 832-837.

[Scollo et al., 2009] Scollo, S., Prestifilippo, M. , Spata, G., D'Agostino, M. and Coltelli M. (2009). Forecasting and monitoring Etna volcanic plumes, Nat. Hazards Earth Syst. Sci., 9, 1573-1585; doi:10.5194/nhess-9-1573-2009.

[Scollo et al., 2014] Scollo S., Prestifilippo, M. et al. (2014). Eruption column height estimation of the 2011-2013 Etna lava fountains, Annals of Geophysics, 57, doi:10.4401/ag-6396. 